\title{
Création et sélection de populations diploïdes de pomme de terre (Solanum tuberosum $L$ )
}

\author{
F Rousselle-Bourgeois, P Rousselle \\ INRA, station d'amélioration de la pomme de terre et des plantes à bulbes, Kéraiber 29260 Ploudaniel, France
}

(Reçu le 9 mai 1991; accepté le 25 novembre 1991)

\begin{abstract}
Résumé - Un grand nombre ( 3027$)$ de clones dihaploïdes $(2 n=2 x=24)$ a été extrait de 445 clones de pomme de terre tétraploïde $(2 n=4 x=48)$ par parthénogénèse in situ. Les résultats de ces pollinisations ont été de l'ordre de 2-3 graines par baie parmi lesquelles $27 \%$ étaient dihaploïdes. Les clones obtenus sont caractérisés par une faible vigueur, une stérilité mâle généralisée, mais expriment une grande variabilité quant à l'aspect de la végétation, les caractéristiques des tubercules, les caractères technologiques (teneur en matière sèche et aptitude à la friture) et les résistances. Ils ont été hybridés avec 4 espèces apparentées diploïdes (S phureja, $S$ stenotomum, $S$ chacoense, $S$ vernei). Les hybrides de $1^{\text {re }}$ génération sont recroisés entre eux ou sur d'autres dihaploïdes, puis différentes combinaisons sont réalisées tout en maintenant les 4 groupes séparés selon l'espèce introduite au départ. Les hybrides montrent une vigueur importante et une fertilité mâle suffisante bien que celle-ci dépende du clone dihaploïde parent. Ils sont aussi caractérisés pour des facteurs tels que teneur en matière sèche et aptitude à la transformation en frites. Ces populations diploïdes améliorées, adaptées aux conditions de culture françaises et présentant une importante variabilité, servent de base à des programmes ultérieurs de retour au niveau tétraploöde, d'utilisation des biotechnologies et d'introduction de nouvelles espèces apparentées.
\end{abstract}

Solanum tuberosum = pomme de terre / amélioration génétique / diploïde / espèces apparentées

Summary - Creation and breeding of diploid populations in potato (Solanum tuberosum $L$ ). A large number ( 3 027) of dihaploids $(2 n=2 x=24)$ has been obtained from 445 tetraploid $(2 n=4 x=48$ ) potato clones by means of in situ parthenogenesis. These pollinations gave about 2 to 3 seeds per fruit. Of the seedlings $27 \%$ were dihaploid. The dihaploids had weak vigour and were all male sterile, but showed a wide variability for plant aspect, tuber characteristics, processing quality (dry matter content and ability to be fried) and disease and pest resistance. They were subsequently included in hybridizations with 4 diploid related species $(S$ phureja, $S$ stenotonum, $S$ chacoense and $S$ vernei). First generation hybrids were intercrossed or crossed with other dihaploids and different combinations were then obtained. Hybrid groups were distinguished depending on the species used. These hybrids were very vigourous and exhibited enough male fertility, the latter depending on the dihaploid used. They were described for characters such as dry matter content and chipping quality. The advanced diploid populations prove to be adapted to French cropping conditions and show interesting variability. They constitute a basis for the resynthesis of tetraploid clones and they will be used for the application of biotechnology and the introduction of new related species.

Solanum tuberosum $=$ potato $/$ breeding $/$ diploid $/$ related species

\section{INTRODUCTION}

La pomme de terre cultivée, Solanum tuberosum $L$, est une espèce tétraploïde $(2 n=4 x=$ 48) dont les cultivars sont des clones hétérozygotes. Le schéma classique d'amélioration génétique repose sur le croisement de 2 parents choisis en valeur propre pour leurs caractères complémentaires, puis la recherche dans la descendance des individus les meilleurs qui sont maintenus par multiplication végétative. Cette méthode a conduit à la création d'un grand nombre de variétés. Elle est cependant limitée par l'hérédité tétrasomique de la plupart des ca- ractères, par l'hétérozygotie des parents et par la base génétique relativement étroite, due au faible nombre de clones introduits au départ à partir d'Amérique du Sud, région d'origine.

En 1963, Chase a proposé un schéma de sélection analytique devant théoriquement permettre de faire de nouveaux progrès et basé sur les 3 étapes suivantes:

- diploïdisation des cultivars tétraploïdes;

- sélection au niveau $2 x$,

- retour au niveau tétraploïde (doublement du nombre de chromosomes par traitement à la colchicine, suivi de croisements sexués ou, plus hy- 
pothétiquement, par hybridation de clones $2 x$ produisant des diplogamètes).

La première étape est rendue possible grâce au phénomène de parthénogénèse induite par pollinisation des clones tétraploïdes par certains clones de Solanum phureja Juz et Buk (Hougas et al, 1958). Les clones obtenus sont appelés dihaploïdes puisqu'ils correspondent à la phase haploïde de l'espèce cultivée mais au niveau diploïde du groupe d'espèces. Cette obtention de dihaploïdes peut être intégrée dans un programme de sélection (Hougas et al, 1964; Hermsen et Verdenius, 1973).

La sélection au niveau diploïde permet de tirer avantage de ségrégations génétiques plus simples et de l'introduction des espèces apparentées diploïdes. Elle aboutit à des clones diploïdes présentant des caractères favorables à l'état hétérozygote.

Le retour au niveau tétraploïde est indispensable car même les meilleurs diploïdes ne remplissent par les exigences d'une variété commerciale (en particulier au niveau productivité). Il est, actuellement, envisagé par 3 techniques :

- doublement du nombre de chromosomes par culture in vitro (Karp et al, 1984; Sonnino et al, 1988; Rousselle et al, 1990);

- fusion de protoplastes (Wenzel et al, 1979);

- polyploïdisation sexuelle grâce aux diplogamètes (Mendiburu et Peloquin, 1977).

Cet article a pour objectif de faire le bilan de près de 20 ans de sélection au niveau diploïde par la mise en œuvre des 2 premières étapes du schéma de Chase. Le but de ce travail était la création de populations diploïdes présentant une bonne adaptation générale aux conditions climatiques de la France et des caractéristiques du tubercule se rapprochant de celles des cultivars tout en ayant une nouvelle base génétique grâce à l'apport des espèces apparentées. Quatre d'entre elles ont été utilisées parmi les diploïdes les plus proches et ne posant pas de problèmes d'hybridation : 2 espèces cultivées Solanum phureja Juz et Buk et Solanum stenotomum Juz et Buk susceptibles d'apporter d'assez bons caractères agronomiques et 2 espèces sauvages, Solanum vernei Bitt et Wittm choisie spécifiquement pour sa résistance au nématode, et Solanum chacoense Bitt qui apporte une grande vigueur. Les 3 premières appartiennent à la série tuberosa des Solanum tubéreuses et la quatrième à la série yungasensa, de plus $S$ stenotomum est un ancêtre probable de $S$ tuberosum (Hawkes, 1990).

\section{MATÉRIEL ET MÉTHODES}

\section{Matériel végétal}

Les dihaploïdes $(\mathrm{DH})$ sont obtenus à partir de variétés en collection et de clones sélectionnés au laboratoire. Ceci représente un échantillonnage du matériel tétraploïde européen. Deux clones de Solanum phureja ont été utilisés comme inducteurs de dihaploïdes : WRF 1203.4 (Université du Wisconsin, USA) et IvP 48 (IVP Wageningen, NL).

Les 4 espèces apparentées diploïdes, $S$ phureja (phu), S stenotomum (stn), $S$ chacoense (chc) et $S$ vernei (vrn) ont été introduites sous forme de familles de graines fournies par la station de Sturgeon Bay (Wisconsin, USA). Le numéro PI est le code d'identification de la famille dans la collection d'origine. Elles sont ensuite maintenues sous forme de quelques clones. Comme elles présentent la caractéristique défavorable d'être adaptées à la tubérisation en jours courts, elles doivent être multipliées à contre saison dans nos conditions. Les génotypes utilisés n'ont pas été analysés pour des caractères précis tels que des résistances.

\section{Obtention des dihaploïdes}

Les clones tétraploïdes sont pollinisés par $S$ phureja après castration. La plupart des pollinisations sont faites sur tiges florales provenant de plantes cultivées au champ et conservées en vase (Hougas et Peloquin, 1959). Les graines ainsi obtenues sont semées en serre. Elles donnent un mélange de plantes diploïdes issues du développement parthénocarpique de l'oosphère et de plantes tétraploïdes hybrides issues de la fécondation d'un ovule tuberosum par un grain de pollen non réduit de phureja. La possibilité de tri des graines contenant un embryon tétraploïde hybride grâce à la tache embryonnaire de IVP 48 (Hermsen et Verdenius, 1973) n'a pas été exploitée : compte tenu du petit nombre de graines, il est rapide et sûr de semer l'ensemble des graines et de contrôler le niveau de ploïdie des plantules. Le niveau de ploïdie est estimé par comptage de chloroplastes sur l'épiderme des feuilles (Frandsen, 1968) et par observation morphologique des plantes pour éliminer les plantes tétraploïdes hybrides. Les tubercules des individus présumés diploïdes sont plantés l'année suivante au champ où ils sont sélectionnés pour leur tubérisation. Le nombre de chromosomes est vérifié sur pointes de racines pour les clones sélectionnés. Les clones conservés sont ensuite analysés pour un certain nombre de caractéristiques comme décrit ci-dessous.

\section{Hybridation avec les espèces apparentées}

Compte tenu du matériel utilisé qui est très proche du point de vue taxonomique, ces hybridations sont réali- 
sées comme des croisements intraspécifiques sur des plantes cultivées en serre ou tunnel plastique.

\section{Sélection des hybrides diploïdes}

Les graines sont semées en serre et la croissance des plantes est arrêtée par suppression de l'arrosage au bout d'environ $120 \mathrm{j}$ de végétation. Les individus conservés sont plantés au champ l'année suivante à raison d'un tubercule par clone; chaque famille de pleinfrères est identifiée. Le clonage intervient dès la première année au champ ou parfois la deuxième année. La sélection porte ensuite sur la vigueur, la précocité, la floraison, la fertilité mâle, l'aspect du tubercule. Éventuellement, des essais permettent d'évaluer le rendement et des caractéristiques technologiques. Les clones sont maintenus par 8 tubercules.

\section{Évaluation des caractéristiques des clones diploïdes}

La fertilité mâle est estimée par coloration au carmin acétique du pollen de fleurs prélevées au champ.

Le rendement en tubercules est mesuré sur des parcelles de 5 ou 6 plantes récoltées à maturité. La teneur en matière sèche est évaluée par la mesure de la densité des tubercules selon la méthode de von Scheele et al (1937) sur des échantillons de 1-5 kg. L'aptitude à la transformation en frites est appréciée sur 30 frites tirées de 30 tubercules par clone qui sont classées en 3 classes selon l'intensité de leur coloration (claires, moyennes et foncées), les clones sont comparés par leur pourcentage de frites à coloration (claire + moyenne).

La résistance auX virus $X$ et $Y$ (hypersensibilité ou résistance extrême) est testée en serre après inoculation par greffage ou frottement (Cornuet, 1987). La résistance à Globodera rostochiensis Woll (gène $\mathrm{H} 1$ ) est analysée par tests en pots (Mugniéry et Balandras, 1986).

\section{RÉSULTATS}

\section{Sélection des dihaploides}

\section{Obtention}

Entre 1967 et 1987, 3037 dihaploïdes ont été extraits de 445 clones tétraploïdes. Le nombre de graines par baie n'a pas été évalué précisément faute du décompte des fruits contenant des graines. II est de l'ordre de 2-3, alors qu'il peut atteindre 200 pour des croisements entre clones tétraploïdes. Le tableau I récapitule les résultats
Tableau I. Résultats des extractions de dihaploïdes selon l'inducteur et le clone $4 x$ femelle (1967-1987).

\begin{tabular}{|c|c|c|c|c|}
\hline & \multirow{2}{*}{$\begin{array}{c}N b \\
\text { graines } \\
\text { semées }\end{array}$} & \multirow{2}{*}{$\begin{array}{c}N b \\
\text { plantes }\end{array}$} & \multicolumn{2}{|c|}{ Dihaploïdes } \\
\hline & & & $N b$ & $1100 \mathrm{pl}$ \\
\hline \multicolumn{5}{|l|}{ Pollinisateur } \\
\hline WRF 12034 & 9726 & 5262 & 1151 & 22 \\
\hline IvP 48 & 13694 & 5991 & 1886 & 31 \\
\hline Total & 23420 & 11253 & 3037 & 27 \\
\hline \multicolumn{5}{|l|}{ Clone femelle } \\
\hline Ajax & 203 & 110 & 73 & 66 \\
\hline Aminca & 183 & 97 & 27 & 28 \\
\hline Claustar & 352 & 165 & 73 & 44 \\
\hline Juliver & 338 & 148 & 15 & 12 \\
\hline Lizen & 852 & 413 & 164 & 40 \\
\hline
\end{tabular}

des pollinisations de 1967 à 1987. Le taux de germination des graines est de $48 \%$ avec des variations très importantes selon l'année (13$86 \%$ ). En moyenne, $27 \%$ des plantules sont des dihaploïdes. L'inducteur IVP 48 donne de meilleurs résultats. II existe une grande variation selon le clone femelle ainsi que le montrent les quelques exemples donnés (tableau I).

\section{Caractérisation}

Les dihaploïdes sont peu vigoureux. Certains ne tubérisent pas en conditions de culture au champ. Leur productivité est réduite avec toutefois des niveaux très variables (tableau II).

La caractéristique la plus intéressante pour le sélectionneur est l'expression d'une très grande variabilité : les dihaploïdes mettent en évidence des caractères qui n'apparaissent pas chez les clones tétraploïdes en ce qui concerne la forme et la couleur des tubercules (fig 1), le port et l'aspect du feuillage. Pour la teneur en matière sèche, l'ensemble des dihaploïdes montre une grande dispersion (fig 2) et ils peuvent avoir des valeurs inférieures et supérieures à celle du clone $4 x$ d'origine avec une moyenne du même ordre (tableau III). De même, pour la valeur de transformation en frites, la dispersion est importante (fig 2, tableau III) et la plupart des séries de dihaploïdes - issus d'un même clone $4 x$ testées présentent des clones meilleurs que leur parent.

Les dihaploïdes, issus de clones $4 x$ présentant des résistances aux virus ou à Globodera 
Tableau II. Rendement brut des clones diploïdes de type dihaploïde (DH) et hybrides des groupes d'origine $S$ phureja (phu) et $S$ stenotomum (stn) par rapport à des témoins tétraploïdes.

\begin{tabular}{|c|c|c|c|c|c|c|}
\hline \multirow{3}{*}{$\begin{array}{l}\text { Type } \\
\text { de clones }\end{array}$} & \multicolumn{3}{|c|}{1984} & \multicolumn{3}{|c|}{1985} \\
\hline & \multirow[t]{2}{*}{$N b$} & \multicolumn{2}{|c|}{ Rendement } & \multirow[t]{2}{*}{$N b$} & \multicolumn{2}{|c|}{ Rendement } \\
\hline & & Moy & Extr & & Moy & Extr \\
\hline $\mathrm{DH}$ & 61 & 51 & $7-100$ & 39 & 51 & $30-91$ \\
\hline $\mathrm{DH}^{*}$ phu & 9 & 83 & $66-100$ & 16 & 90 & $57-116$ \\
\hline$\left(\mathrm{DH}^{*} \mathrm{phu}\right)^{2}$ & 6 & 66 & $49-82$ & 6 & 66 & $45-88$ \\
\hline $\mathrm{DH}^{*}\left(\mathrm{DH}^{*} \mathrm{phu}\right)$ & 11 & 72 & $55-87$ & 18 & 70 & $37-103$ \\
\hline$\left[\mathrm{DH}^{*}\left(\mathrm{DH}^{\star} \mathrm{phu}\right)\right]^{*}$ phu & & & & 11 & 76 & $45-111$ \\
\hline$\left[\mathrm{DH}^{*}\left(\mathrm{DH}^{*} \mathrm{phu}\right)\right]^{*}\left(\mathrm{DH}^{*} \mathrm{phu}\right)$ & & & & 16 & 70 & $44-87$ \\
\hline $\mathrm{DH}^{*} \operatorname{stn}$ & 12 & 73 & $39-99$ & 7 & 74 & $44-96$ \\
\hline $\mathrm{DH}^{*}\left(\mathrm{DH}^{*} \mathrm{stn}\right)$ & 17 & 66 & $31-84$ & 13 & 81 & $66-105$ \\
\hline
\end{tabular}

$\mathrm{Nb}=$ nombre de clones; Moy = moyenne; Extr = valeurs extrêmes; rendements exprimés en \% de (Bintje+Claustar) $/ 2 ; 1984: 10,9 \pm 0,9$ $\mathrm{kg}=100 \pm 9$ pour 5 plantes (moyenne sur 10 parcelles); $1985: 9,1 \pm 1,1 \mathrm{~kg}=100 \pm 12$ pour 5 plantes (moyenne sur 12 parcelles).

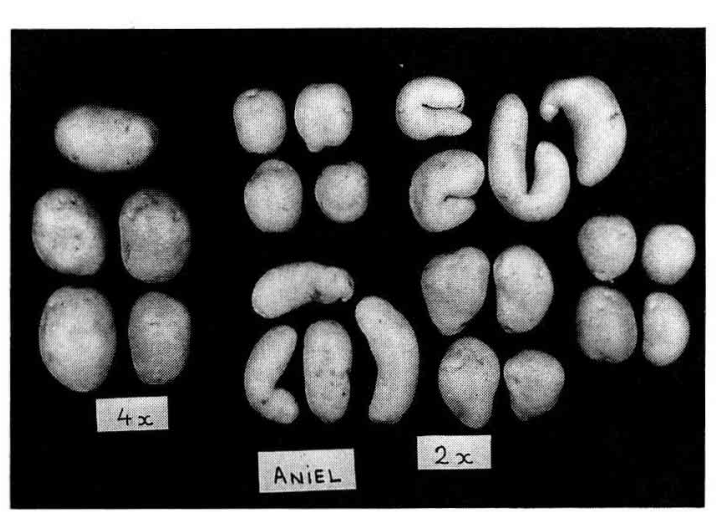

Fig 1. Tubercules de 5 clones dihaploïdes (4 tubercules de chaque) issus de la variété Aniel en comparaison avec ceux du tétraploïde d'origine (5 tubercules).

rostochiensis, ont été testés pour celles-ci. Pour les différents parasites, environ $50 \%$ des clones se montrent résistants (tableau IV). Une analyse des ségrégations n'est pas possible car les tests ont été pratiqués seulement sur les clones ayant subi une sélection préalable pour la tubérisation.

Les dihaploïdes sont mâle stériles dans leur grande majorité. Le tableau $V$ illustre ce phénomène sur un échantillon et pour une année donnée qui représentent le cas général. Seuls les clones ayant un pourcentage de pollen bien coloré supérieur à $50 \%$ peuvent être utilisés comme parents mâles. Ces résultats montrent qu'il n'est pas possible d'envisager un programme de croisements et de sélection au sein du groupe tuberosum diploïde. Par contre, la fertilité femelle est peu ou pas affectée pour les clones qui sont aptes à fleurir.
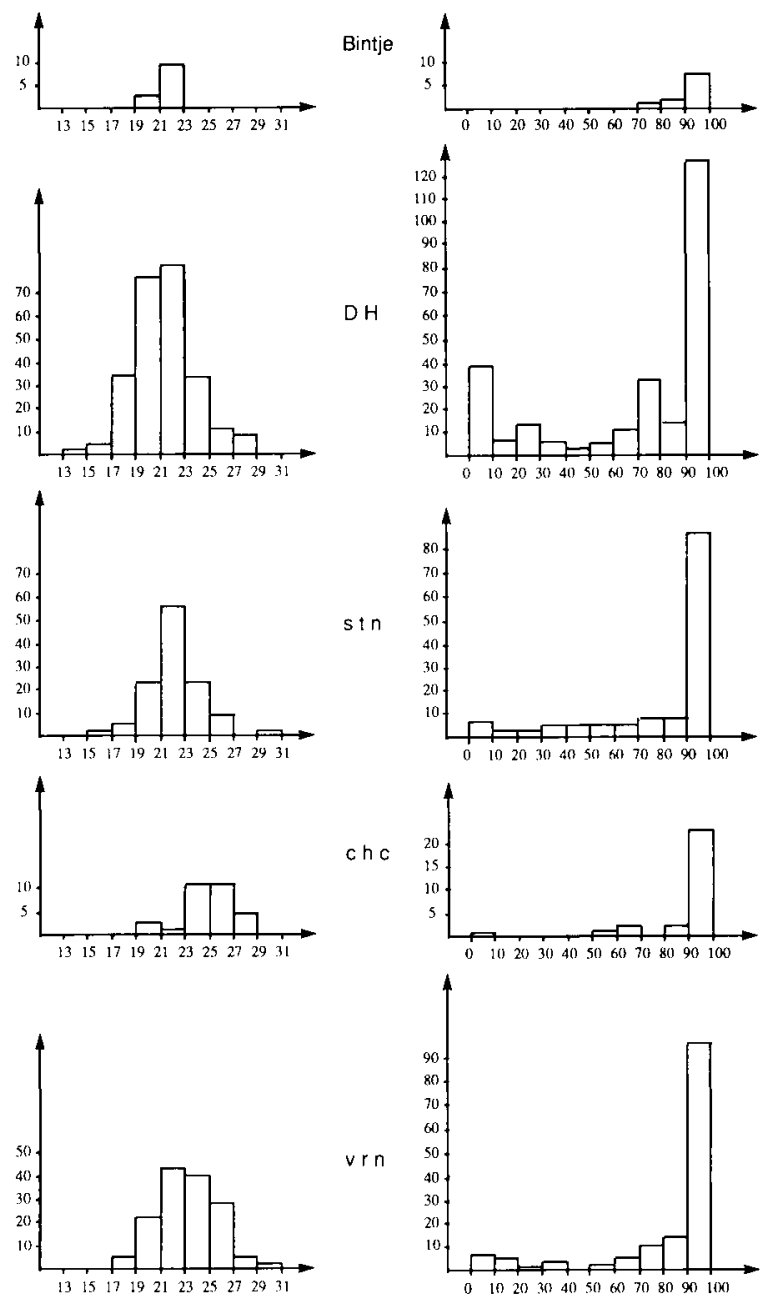

Fig 2. Teneur en matière sèche et aptitude à la transformation en frites de différents groupes de diploïdes. Ordonnée : nombre de clones; abscisses : matière sèche exprimée en \% (à gauche), aptitude à la transformation en frites exprimée en $\%$ de frites acceptables (à droite). $\mathrm{DH}$ : dihaploïdes tuberosum; stn : hybrides avec $S$ stenotomum; chc : hybrides avec $S$ chacoense; vrn : hybrides avec $S$ vernei. 
Tableau III. Teneur en matière sèche et aptitude à la transformation en frites de 9 variétés tétraploïdes et des dihaploïdes qui en sont issus.

\begin{tabular}{|c|c|c|c|c|}
\hline \multirow{2}{*}{$\begin{array}{l}\text { Variété } \\
\text { d'origine }\end{array}$} & \multirow{2}{*}{$\begin{array}{l}\text { Valeur } \\
\text { variété }\end{array}$} & \multicolumn{3}{|c|}{ Clones dihaploïdes } \\
\hline & & $N b$ & Moy & Extr \\
\hline Fanette & $\begin{array}{l}17,8^{a} \\
20^{b}\end{array}$ & 4 & 18,4 & $\begin{array}{c}16,6-19,9 \\
0-100\end{array}$ \\
\hline Claustar & $\begin{array}{l}18,7 \\
13\end{array}$ & 8 & 18,5 & $\begin{array}{l}17,5-20,1 \\
10-100\end{array}$ \\
\hline Monalisa & $\begin{array}{l}19,8 \\
50\end{array}$ & 6 & 19,0 & $\begin{array}{c}15,6-21,5 \\
0-100\end{array}$ \\
\hline Aminca & $\begin{array}{l}21,2 \\
80\end{array}$ & 10 & 22,0 & $\begin{aligned} 19,7 & -24,5 \\
100 & -100\end{aligned}$ \\
\hline Dani & $\begin{array}{l}21,3 \\
100\end{array}$ & 3 & 20,6 & $\begin{array}{l}18,6-22,3 \\
97-100\end{array}$ \\
\hline Lizen & $\begin{array}{l}21,4 \\
87\end{array}$ & 36 & 20,5 & $\begin{array}{c}16,3-23,9 \\
0-100\end{array}$ \\
\hline Nicola & $\begin{array}{c}21,8 \\
0\end{array}$ & 8 & 20,9 & $\begin{array}{c}16,7-23,3 \\
0-73\end{array}$ \\
\hline Cardinal & $\begin{array}{l}22,1 \\
80\end{array}$ & 7 & 22,7 & $\begin{array}{l}21,5-25,4 \\
90-100\end{array}$ \\
\hline Astarte & $\begin{array}{l}25,8 \\
100\end{array}$ & 8 & 25,8 & $\begin{array}{l}22,2-28,6 \\
70-100\end{array}$ \\
\hline
\end{tabular}

$a$ : teneur en matière sèche; $b: \%$ de frites (claires + moyennes). $N b=$ nombre de dihaploïdes analysés; Moy = moyenne; Extr = valeurs extrêmes.

Tableau IV. Résultats des tests de résistance aux virus $\mathrm{X}, \mathrm{Y}, \mathrm{A}$ et à Globodera rostochiensis sur des clones dihaploïdes.

$$
D H^{N b} \operatorname{Var} \quad S \quad R
$$

\begin{tabular}{lrrrr}
\hline & & & & \\
Virus X & 135 & 16 & 65 & 70 \\
Virus Y & 26 & 3 & 11 & 15 \\
Virus A & 122 & 25 & 58 & 64 \\
G rostochiensis & 110 & 19 & 51 & 59 \\
\hline
\end{tabular}

$D H$ : dihaploïdes testés; Var : clones $4 x$ représentés; $S$ : nombre de sensibles; $R$ : nombre de résistants.

Les critères de sélection des dihaploïdes ont été : aptitude à tubériser, vigueur suffisante pour être maintenus, tubercules sans défauts graves, origines génétiques les plus diverses possibles
Tableau V. Fertilité pollinique des dihaploïdes estimée par coloration au carmin acétique.

\% pollen coloré Nb de clones a

\begin{tabular}{lr}
\hline Étaminés avortées & 26 \\
0 & 117 \\
$1-20$ & 26 \\
$21-50$ & 16 \\
$>50$ & 1 \\
\hline
\end{tabular}

a 186 clones ayant fleuri au champ en 1985.

pour conserver une large variabilité qui est un des buts de ce programme. Globalement, la pression de sélection a tendance à être moins forte pour les séries où il y a peu de dihaploïdes.

\section{Sélection des diploïdes hybrides}

\section{Obtention}

La première étape est l'obtention d'hybrides interspécifiques entre un dihaploïde et un clone d'une espèce apparentée ( $\left.\mathrm{DH}^{*} \mathrm{sp}\right)$. Pour améliorer l'adaptation et l'aspect des tubercules, les hybrides de $1^{\text {re }}$ génération sont recroisés entre eux $\left(\mathrm{DH}^{*} \mathrm{sp}\right)^{2}$ ou sur un dihaploïde $\left[\mathrm{DH}^{*}\right.$ $\left.\left(\mathrm{DH}^{*} \mathrm{sp}\right)\right]$, ensuite différentes combinaisons de ces types de clones sont réalisées. Dans la mesure du possible, les clones entrant dans une combinaison sont choisis avec des origines génétiques différentes, toutefois cette pratique théorique peut être biaisée par les problèmes de fertilité qui limitent les possibilités de choix.

Les groupes de clones ont été maintenus séparés en fonction de l'espèce apparentée introduite (pas d'intercroisement entre clones hybrides avec $S$ phureja et $S$ chacoense par exemple), ceci afin de mieux cerner l'intérêt de chaque espèce.

Environ $1 / 3$ des plantes issues de semis sont conservées (tableau VI). Selon les types d'hybrides, de 3 à $12 \%$ des plantes issues de semis ne tubérisent pas en serre. La sélection tient compte de la précocité et des caractéristiques du tubercule (forme, profondeur des yeux, couleur). Les taux de sélection sont du même ordre de grandeur pour les différents types mais les critères sont plus sévères pour les hybrides de $2^{\theta}$ génération ou ultérieure. 
Tableau VI. Taux de sélection des semis et en première année au champ des hybrides de 1981 à 1986.

\begin{tabular}{|c|c|c|c|c|c|}
\hline \multirow[t]{2}{*}{ Type d'hybride } & \multicolumn{3}{|c|}{ Serre } & \multicolumn{2}{|c|}{ Champ } \\
\hline & Nb semés & $\% N T$ & \% gardés & Nb plantés & \% gardés \\
\hline chc ensemble & 2976 & 6 & 35 & 1023 & 18 \\
\hline $\mathrm{DH}^{*}$ phu & 15403 & 4 & 36 & 5185 & 38 \\
\hline$\left(\mathrm{DH}^{*} \mathrm{phu}\right)^{2}$ & 14207 & 9 & 42 & 5207 & 39 \\
\hline$\left(\mathrm{DH}^{*} \mathrm{phu}\right)^{3}$ & 2155 & 11 & 39 & 814 & 37 \\
\hline$\left(\mathrm{DH}^{*} \mathrm{phu}\right)^{2^{*} \mathrm{phu}}$ & 7197 & 12 & 19 & 1231 & 35 \\
\hline $\mathrm{DH}^{*}\left(\mathrm{DH}^{*} \mathrm{phu}\right)$ & 3923 & 7 & 36 & 1214 & 23 \\
\hline$\left[\mathrm{DH}^{\star}\left(\mathrm{DH}^{\star} \text { phu }\right)\right]^{\star}\left(\mathrm{DH}^{\star} \text { phu }\right)^{2}$ & 6376 & 5 & 30 & 1785 & 33 \\
\hline phu ensemble & 61241 & 8 & 32 & 17847 & 35 \\
\hline $\mathrm{DH}^{*} \operatorname{stn}$ & 7557 & 10 & 28 & 2029 & 33 \\
\hline$\left(\mathrm{DH}^{*} \operatorname{stn}\right)^{2}$ & 4057 & 10 & 38 & 1431 & 39 \\
\hline $\mathrm{DH}^{*}\left(\mathrm{DH}^{*} \mathrm{stn}\right)$ & 1610 & 6 & 53 & 819 & 19 \\
\hline$\left[\mathrm{DH}^{*}\left(\mathrm{DH}^{*} \mathrm{stn}\right)\right]^{*}\left(\mathrm{DH}^{*} \operatorname{stn}\right)$ & 1787 & 8 & 31 & 548 & 68 \\
\hline $\mathrm{DH}^{*}\left[\mathrm{DH}^{*}\left(\mathrm{DH}^{*} \mathrm{stn}\right)\right]$ & 1867 & 3 & 43 & 747 & 21 \\
\hline stn ensemble & 19612 & 9 & 33 & 6126 & 35 \\
\hline
\end{tabular}

$N T=$ non tubérisés; chc : $S$ chacoense; phu : $S$ phureja; $\operatorname{stn}: S$ stenotomum.

À l'issue de la $1^{\text {re }}$ année au champ, $35 \%$ des plantes d'origine phu et stn sont conservées. Pour les plantes d'origine chc, ce pourcentage n'est que de $18 \%$. II s'explique par le fait que l'adaptation à nos conditions de ces clones est nettement moins bonne et qu'ils tubérisent mal. Les taux de sélection pour les origines vrn ne sont pas portés dans le tableau puisque ces clones subissent un tri spécifique pour la résistance à Globodera pallida qui ne sera pas discutée ici (Rousselle et al, 1989).

\section{Caractérisation}

La caractéristique la plus évidente des hybrides de $1^{\text {re }}$ génération est leur grande vigueur, souvent liée à une tardivité importante apportée par l'espèce apparentée. Ceci se traduit par une productivité très supérieure à celle des dihaploïdes (tableau II). Les hybrides d'origine phu issus de recroisements ou d'intercroisements ont tendance à être moins vigoureux que ceux de première génération. Par contre, pour stn, dans l'essai de 1985, les hybrides de type $\left[\mathrm{DH}^{*}\right.$ $\left.\left(\mathrm{DH}^{*} \mathrm{stn}\right)\right]$ ont été en moyenne plus productifs que les hybrides de première génération $\left(\mathrm{DH}^{*} \mathrm{stn}\right)$ alors que la tendance était inverse en 1984. Ces essais n'avaient qu'un but indicatif sur la valeur propre des diploïdes; il n'est pas possible de comparer les résultats d'une année à l'autre puisque les clones testés ne sont pas les mêmes. Les hybrides avec chc et vrn n'ont pas été évalués en essais de rendement en raison de leur trop grande tardivité.

Dans tous ces hybrides, on retrouve les aspects des tubercules des espèces apparentées: couleurs variées (blanc, jaune, rouge, violet, uniforme ou non), yeux plus ou moins enfoncés, formes irrégulières et tourmentées (fig 3). II est cependant possible de trouver des clones ayant les caractéristiques de $S$ tuberosum, en particulier dans les hybrides de deuxième génération et ultérieures. Ces clones ont été sélectionnés en priorité.

Les mesures de la teneur en matière sèche et de l'aptitude à la transformation en frites ont été

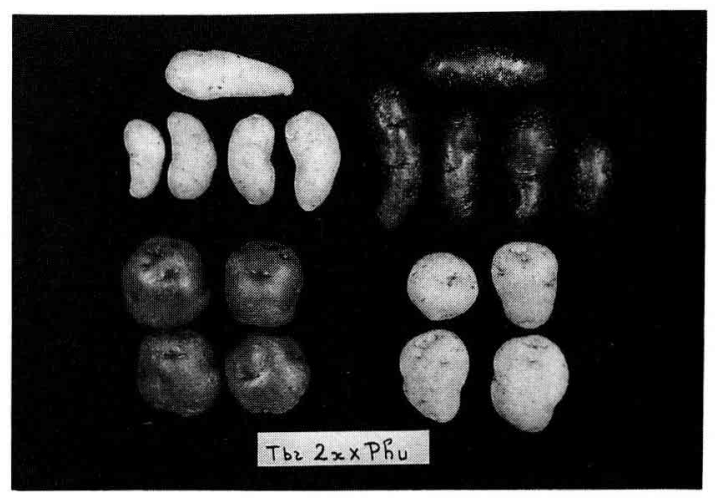

Fig 3. Tubercules de 4 clones diploïdes hybrides de type $\mathrm{DH}^{*}$ phu (5 ou 4 tubercules de chaque); phu : $S$ phureja. 
réalisées sur les groupes stn, chc et vrn. Les hybrides avec phu n'ont pas été analysés dans un premier temps car cette espèce ne donne pas de bonnes teneurs et sa mauvaise aptitude à la conservation de longue durée ne l'oriente pas vers la création de géniteurs de type transformation industrielle. La figure 2 illustre une importante dispersion pour ces 2 caractères avec la présence de clones à forte teneur en matière sèche et à bonne aptitude à la friture.

La fertilité mâle des hybrides de première génération est variable selon les familles, elle dépend en particulier de la femelle dihaploïde utilisée (tableau VII). Certains dihaploïdes ne donnent pas de descendances fertiles (73.51.33 $\mathrm{H} 1$, Famosa $\mathrm{H} 1$, U.LANCER H1 ou DIAMANT $\mathrm{H} 2$ ). II peut exister une interaction entre les dihaploïdes et les clones d'espèce apparentée mais la structure des croisements ne permet pas de l'analyser. Toutefois, la fertilité mâle est suffisamment améliorée pour que la poursuite de la création de nouveau matériel soit envisagée. II n'est pas apparu de problème majeur de fertilité femelle dans ce type de matériel.

\section{DISCUSSION}

Les taux de réussite des pollinisations d'extraction de dihaploïdes sont du même ordre de grandeur que ceux des auteurs cités précédemment (Hougas et al, 1964; Hermsem et Verdenius, 1973) : le rendement en dihaploïdes est faible et il existe une forte influence du parent femelle. Le taux de germination de $48 \%$ peut être expliqué par le fait que les fruits ont été obtenus sur inflorescence en vase; leur grossissement est moins bon que sur plante entière. Les différences importantes constatées entre les années peuvent s'expliquer par l'effet de l'année d'obtention (état physiologique des plantes mères, conditions en serre), par l'effet des conditions de semis et par l'effet des génotypes qui sont différents chaque année. Le succès est suffisant pour que cette technique ait été intégrée dans le travail de sélection.

La mesure du rendement brut en tubercules des clones diploïdes donne une évaluation de leur vigueur et de leur capacité à produire des tubercules. La comparaison avec les clones tétraploïdes serait encore plus en défaveur des diploïdes si les rendements commerciaux mesurés après calibrage étaient comparés. En effet les tubercules produits par les diploïdes sont souvent nombreux mais petits.

Les teneurs en matière sèche et l'aptitude à la transformation en frites mesurées sur les dihaploïdes vont dans le même sens que les observations de Kotch (1987) sur la densité et la teneur en sucres réducteurs qui sont liées respectivement aux 2 caractères que nous avons analysés : la variabilité exprimée est large et certains dihaploïdes sont meilleurs que le tétraploïde d'origine.

Les clones diploïdes hybrides ont été sélectionnés pour leur aptitude à la floraison afin

Tableau VII. Pourcentage de plantes mâle fertiles dans des descendances de type $\mathrm{DH}^{\star}$ phu et $\mathrm{DH}^{\star}$ stn.

\begin{tabular}{|c|c|c|c|c|c|c|c|}
\hline $\begin{array}{l}\text { Parent } \\
D H\end{array}$ & $\begin{array}{c}\text { phu } \\
P I \\
225681\end{array}$ & $\begin{array}{c}\text { phu } \\
P I \\
195198\end{array}$ & $\begin{array}{c}\text { phu } \\
P I \\
225672\end{array}$ & $\begin{array}{c}\text { phu } \\
P I \\
243464\end{array}$ & $\begin{array}{c}s t n \\
P l \\
234013\end{array}$ & $\begin{array}{c}\text { stn } \\
P l \\
195304\end{array}$ & $\begin{array}{c}\text { stn } \\
P l \\
458393\end{array}$ \\
\hline $57.26 .11 \mathrm{H} 1$ & 79 & 81 & 39 & 88 & & & \\
\hline $57.23 .11 \mathrm{H} 2$ & - & 77 & - & 88 & & & \\
\hline 73.48.39 H5 & - & 88 & 100 & - & & & \\
\hline 73.51.33 H1 & 0 & - & 2 & - & & & \\
\hline Arka H6 & - & 78 & 89 & - & & & \\
\hline Claustar H3 & 22 & 5 & - & - & & & \\
\hline Famosa H1 & 0 & - & 0 & - & & & \\
\hline Lizen H2 & 6 & 9 & 10 & - & & & \\
\hline U Lancer H1 & 0 & 0 & - & - & & & \\
\hline Jose H3 & & & & & 39 & 30 & - \\
\hline Diamant H2 & & & & & 0 & 0 & 0 \\
\hline Lori H6 & & & & & 20 & 13 & - \\
\hline M Page H1 & & & & & - & 69 & 40 \\
\hline
\end{tabular}

Fertilité estimée par coloration du pollen au carmin acétique; phu : $S$ phureja; $\operatorname{stn}: S$ stenotomum. 
d'être utilisés dans des programmes de croisements pour poursuivre le travail d'amélioration. Ceci a pour conséquence d'éliminer les clones ayant une tubérisation précoce et de favoriser les clones tardifs. En effet il y a antagonisme entre la tubérisation et la croissance de la partie aérienne, ce qui explique que les clones les plus précoces fleurissent peu ou mal. II conviendra lors du retour au niveau tétraploïde de contresélectionner ce caractère.

L'ensemble des populations décrites possèdent le cytoplasme de $S$ tuberosum puisque pratiquement les dihaploïdes ne peuvent être utilisés qu'en tant que femelles. Ceci est en contradiction avec la recherche d'une nouvelle variabilité cytoplasmique mais c'est un cas général dans ce type de programme de sélection au niveau diploïde chez la pomme de terre.

\section{CONCLUSION}

En 1990, 463 dihaploïdes représentant 152 tétraploïdes sont présents en collection. À titre de comparaison, l'assortiment de l'université du Wisconsin (USA) comprend environ 400 clones sélectionnés parmi 2000 obtentions et dont une soixantaine sont intéressants (Peloquin com pers). La population d'hybrides est composée de 1018 clones sélectionnés répartis dans les 4 populations et se trouvant à des niveaux divers d'hybridation depuis l'introduction de l'espèce apparentée. Son importance est comparable à celle de la collection de l'Institut de recherche sur la pomme de terre polonais (ZimnochGuzowska et Dziewonska, 1989) et elle est originale par rapport à celle d'autres instituts qui cherchent à utiliser directement les dihaploïdes (Munzert et Scheidt, 1989; De, Maine, 1982).

Afin d'exploiter la variabilité créée, la priorité est à l'heure actuelle l'obtention de clones tétraploïdes particulièrement par utilisation des diplogamètes mâles et doublement chromosomique. L'objectif n'est plus d'obtenir des variétés directement, mais plutôt de créer des populations sources tétraploïdes qui subiront de nouvelles recombinaisons.

Certains clones diploïdes sont utilisés par d'autres laboratoires pour des travaux de fusions de protoplastes (Masson et al, 1989; Simo et Boccon-Gibod, 1989). L'ensemble du matériel caractérisé pourra servir de base à des études de biologie moléculaire qui se développent très rapidement chez la pomme de terre et pour les- quelles le niveau diploïde est plus facile à manipuler. II en est de même pour des analyses génétiques plus traditionnelles (étude de l'héritabilité de certains caractères).

En complément de la valeur agronomique déjà acquise dans ces populations, un nouveau cycle de création de matériel est commencé à partir de nouvelles introductions d'espèces apparentées choisies pour leurs caractères de résistance (maladies bactériennes, fusariose, virus de l'enroulement, nouvelles sources pour les virus $X$ et $Y$ ou les nématodes) ou de valeur technologique (teneur en matière sèche et en sucres réducteurs). II s'appuiera sur le matériel existant et bénéficiera de l'expérience acquise.

\section{REMERCIEMENTS}

Ce programme a été mis en place par $\mathrm{P}$ Perennec et doit beaucoup à la compétence technique de $J$ Le Berre et $J$ François. D Mugniéry a réalisé les tests de résistance à Globodera rostochiensis. Nous remercions $\mathrm{N}$ Robert pour la réalisation de la figure 2.

\section{RÉFÉRENCES}

Chase SS (1963) Analytic breeding in Solanum tuberosum $L$. A scheme utilizing parthenotes and other diploid stocks. Can J Genet Cyto/ 5, 359-363

Cornuet $P$ (1987) Éléments de virologie végétale. INRA, Paris, $206 p$

De Maine MJ (1982) An evaluation of the use of dihaploids and unreduced gametes in breeding for quantitative resistance to potato pathogens. J Agric Sci Camb 99, 79-83

Frandsen NO (1968) Die Plastidenzahl als Merkmal bei der Kartofell. Theor Appl Genet 38, 153-167

Hawkes JG (1990) The potato. Evolution, biodiversity and genetic resources. Belhaven Press, Londres, $259 p$

Hermsen JGTh, Verdenius J (1973) Selection from Solanum tuberosum group Phureja of genotypes combining high-frequency haploid induction with homozygosity for embryo-spot. Euphytica 22, 244-259

Hougas RW, Peloquin SJ, Ross RW (1958) Haploids of the common potato. J Hered 49, 103-106

Hougas RW, Peloquin SJ (1959) Decapitation and genetic markers as related to haploidy in Solanum tuberosum. Eur Potato J 2, 176-183

Hougas RW, Peloquin SJ, Gabert AC (1964) Effect of seeds parent and pollinator on the frequency of haploids in Solanum tuberosum. Crop Sci 4, 593-595

Karp A, Risiott R, Jones MGK, Bright SWJ (1984) Chromosome doubling in monohaploid and diploid 
potatoes by regeneration from cultured leaf explants. Plant Cell Tissue Organ Cult 3, 4, 363-373

Kotch GP (1987) The production of haploids and their use in genetic studies in potatoes. Ph D Thesis, University of Wisconsin (USA), $171 \mathrm{p}$

Masson J, Lancelin D, Bellini C, Lecerf M, Guerche P, Pelletier G (1989) Selection of somatic hybrids between diploid clones of potato (Solanum tuberosum L) transformed by direct gene transfer. Theor Appl Genet 78, 153-159

Mendiburu AO, Peloquin SJ (1977) The significance of $2 n$ gametes in potato breeding. Theor Appl Genet $49,53-61$

Mugniéry D, Balandras C (1986) Test de résistance de descendances de pomme de terre à Globodera rostochiensis Woll. Potato Res 29, 131-140

Munzert M, Scheidt M (1989) Results of a breeding programme with dihaploids of Solanum tuberosum L. In: Parental line breeding and selection in potato breeding (Louwes et al, eds) Pudoc, Wageningen (NL), 163-171

Rousselle P, Balandras C, Mugniéry D (1989) Use of genetic parameters for the assessment of breeding programs for the resistance to Globodra pallida Pa3 (Stone). In: Parental line breeding and selection in potato breeding (Louwes et al, eds) Pudoc, Wageningen (NL), 193
Rousselle P, Rousselle F, François J (1990) Application de la culture in vitro au doublement chromosomique chez la pomme de terre. In: Cinquantenaire de la culture in vitro, Versailles (F), 24-25 oct 1989. Ed INRA, Paris (Les colloques de I'INRA, $n^{\circ}$ 51), 321-323

Simo P, Boccon-Gibod J (1989) Some aspects of protoplast culture and somatic hybridization in potato. In: Parental line breeding and selection in potato breeding (Louwes et al, eds) Pudoc, Wageningen (NL), 199

Sonnino A, Iwanaga M, Henostroza A (1988) Chromosome number doubling of $2 x$ potato lines with diverse genetic background through tissue culture. Potato Res 31, 627-631

von Scheele C, Svensson G, Rasmunsson J (1937) Die Bestimmung des Stärkegehalts und der Trockensubstanz der Kartofell mit Hilfe des spezifischen Gewichts. Landwirtsch Vers Sta 127, 67-96

Wenzel G, Schieder O, Przewozny T, Sopory SK, Melchers G (1979) Comparison of single cell culture derived Solanum tuberosum $L$ plants and a model for their application in breeding programs. Theor App/ Genet 55, 49-55

Zimnoch-Guzowska E, Dziewonska MA (1989) Breeding potato at diploid level. In: Parental line breeding and selection in potato breeding (Louwes et al, eds) Pudoc, Wageningen (NL), 163-171 